\title{
zpl, a new gene in drosophila required for neurogenesis ${ }^{1}$
}

\author{
ZHAO Debiao*, Serge COTE**, Gans JURGENS***, \\ Kavita ARORA ****, Herbert JAECKLE*****. \\ * Shanghai Institute of Cell Biology, Chinese Academy of \\ Sciences, 320 Yoyang Road, Shanghai 200031, China; \\ ** Ontogenese et Genetique Moleculaires, CHUL, Quebec, \\ Canada; \\ *** Institut fur Genetik und Mikrobiologie, Universitat \\ Munchen, Maria-Ward-Str 1A, D-8000 Munchen 19, Ger- \\ many ; \\ **** Max-Planck-Institute of Developmental Biology, Spe- \\ mannstr. 35, D-7400 Tubingen, Germany; \\ ***** Max-Planck-Institut fur Biophysikalische Chemie, Am \\ Fassberg, D-3400 Gottingen, Germany.
}

\begin{abstract}
zpl (zip-like) gene mutant embryos showed the cuticular defect with alternative denticle rows and a hole from head to abdomen. zpl mutants also caused the overgrowth of neural cells and axons both in CNS and PNS as well as the wrong pathway of neural fasciculation and the disappearance of hypophysis, as shown by whole mount embryos stained with antibody against HRP and MAb-22C10. Genetic analysis has provided evidence that $\mathrm{zpl}$, located in the right arm of the second chromosome (between 75 and 102 genetic map units), is a new gene closely related to the zip gene.
\end{abstract}

Key words: zpl, zip, neurogenesis, fasciculation, drosophila.

\section{INTRODUCTION}

During insect neurogenesis, an orthogonal pattern for the nervous system, especiaUy the complex network of neural fascicles, is established in stepwise manner[1,2]. Genetic approaches have led to the identification of a number of genes that have been implicated in these process [3-8, for review]. One of-these genes, zip, has been found

1. The main part of work reported here was finished in Max-Planck-Institute of Developmental Biology, Spemannstr. 35, D-7400 Tubingen, Germany. 
to be involved in the establishment and maintenance of normal neural growth and axon fasciculation. zip encodes a putative integral membrane glycoprotein which may function as an adhesion or recognition cell surface molecule necessary for late neurogenesis of Drosophila [9, 10].

Here a new gene, zpl, is described, which may play a similar important role as zip gene during the Drosophila neurogenesis.

\section{MATERIALS AND METHODS}

\section{Drosophila strains and culture}

zip mutants (ID16, IIF107, IIX62 and ES1, kindly provided by Dr. Nusslein-Volhard) and zpl are all balanced by SM1 or Cyo balancer chromosome. So zip can be maintained as zip/Cyo or zip/ SM1 and zpl as zpl/Cyo. All flies were cultured at $15^{\circ} \mathrm{C}$ or $25^{\circ} \mathrm{C}$ in the following medium: 20 1 water, $160 \mathrm{~g}$ agar, $360 \mathrm{~g}$ dry yeast, $200 \mathrm{~g}$ soya meal, $440 \mathrm{~g}$ molasses, $1600 \mathrm{~g}$ malt extract, $1600 \mathrm{~g}$ maize meal and $125 \mathrm{ml}$ propionic acid ( propionic acid was added after boiling the medium for 10 minutes and cooling it to $60^{\circ} \mathrm{C}$ ).

\section{Genetic mapping}

Males and virgin females were always separated by screening the genitalia for the obvious male genital arch and occasionally also by checking the sex combs. Since zpl gene is carried on the second chromosome, a mutant with five dominant markers (S, Sp, Tft, N-2G, Pu2/Cyo) was used for crossing with the zpl recessive mutant carrying $\mathrm{cn}$ as a mark. The female progeny with straight wings and dominant markers was back crossed. The number of different recombinants among the second progeny generation was determined. The flies with straight wings were carefully examined for genetic markers. This genetic mapping was done according to the protocol in Tab 1.

Tab 1. Protocol of the genetic localization of the $\mathrm{zpl}$ gene

\begin{tabular}{|c|c|c|c|}
\hline $\begin{array}{l}\mathrm{P} \\
\mathrm{F} 1 \\
\mathrm{~F} 2\end{array}$ & $\begin{array}{l}\text { S.Sp.Tft.N-2G.Pu }{ }^{2} / \mathrm{Cyo} \\
\text { cn.sp.bw.zpl/S.Sp.Tft.N-2G.Pu }{ }^{2} \\
\text { cn.sp.bw.zpl/Cyo } \\
\text { S.SP.Tft.N-2G.Pu } / \text { Cyo } \\
\text { cn.sp.bw.zpl/cn. sp.bw.zpl } \\
\text { S.Sp.Tft.N-2G.Pu }{ }^{2} / \text { cn.sp.bw.zpl } \\
\text { RD-zpl/Cyo } \\
\text { RD/Cyo } \\
\text { RD-zpl/cn.sp.bw.zpl } \\
\text { RD/cn.sp.bw.zpl }\end{array}$ & $\begin{array}{l}\mathrm{X} \\
\mathrm{X}\end{array}$ & $\begin{array}{c}\text { cn.sp.bw.zpl/Cyo } \\
\text { cn.sp.bw.zpl/Cyo } \\
\text { curly } \\
\text { curly } \\
\text { die } \\
\text { straight* } \\
\text { curly } \\
\text { curly } \\
\text { die } \\
\text { straight* }\end{array}$ \\
\hline
\end{tabular}

\section{Preparation of the embryonic cuticle}

The embryos (at stage 16-17) were transferred from the agar-plate into a basket with a brush, washed with water and put on paper towels to remove the water. The embryos were dechorionated by transferring the basket into sodium hypochlorite until the slimy surface of vitelline membrane was visible. To remove vitelline membrane, embryos were first sticked on a two-face tesa film sticking on petri dish, using the surface of agar as a medium. The vitelline membrane was popped away from 
the embryos by pressing on the end of embryos with a glass or tungsten needle under microscope. These vitelline membrane-free embryos were transferred into a concave glass and incubated for $1 \mathrm{~h}$ at $60^{\circ} \mathrm{C}$ after adding sufficient glycerol:acetic acid (1:4). Now the embryos could be transferred into a drop of Hoyer's mountant on a glass slide, covered with a coverslip and incubated at $60^{\circ} \mathrm{C}$ until the internal tissue was clear (usually overnight). The cuticle was then examined and photographed under a phase contrast microscope.

\section{Antibody staining of whole mount embryos}

Eggs from zpl/Cyo, zip/Cyo or zip/SM1 matings were collected at 2-h intervals on apple juice/agar plates[11]. After aging at room temperature to the desired developmental stage, embryos were dechorionated, fixed and stained with anti-horseradish peroxidase antibodies (anti-HRP, sigma) according to Jan and Jan[12]. Fixed embryos were preincubated for $3 \mathrm{~h}$ in several changes of phosphate-buffered saline (PBS); $1 \%$ bovine serum albumin (BSA), followed by incubation (overnight, $4^{\circ} \mathrm{C}$ ) in antibody solution (anti-HRP, 1/200 dilution in PBS/0.1\% Triton). After several washes ( $3 \mathrm{~h}$ in total) in $\mathrm{PBS} / 0.1 \%$ Triton, embryos were incubated overnight in peroxidaseconjugated secondary antibodies (Sigma; 1/50 dilution in the same buffer). Embryos were washed as described above, and stained for $2-20 \mathrm{~min}$ in diaminobenzidine $(0.5 \mathrm{mg} / \mathrm{ml}$ in PBS containing hydrogen peoxide). Staining of embryos with neuron-specific antibodies (MAb-22C10, a gift of Dr. A. Ferrus)[13] was performed as described by Gaul et al[14]. After dehydration, embryos were mounted in araldite mounting medium. Stained CNS and PNS of the embryos were analysed at 100- to 400-fold magnification and photographs were taken with Nomarski optics.

\section{RESULTS}

\section{The cuticular phenotype}

One major phenotype of the zpl mutant is a head involution and dorsal closure defect. Therefore, its cuticle often has a big hole from the head to the dorsal side of the abdomen, sometimes combined with a hole in the ventral thoracic region. This was almost the same cuticular phenotype as that of the strong zip mutant as shown in Fig $1 \mathrm{~B}$ and $\mathrm{D}$.

The cuticle of zpl mutant embryos also showed abnormal denticle rows. On the cuticle of normal embryos, there were 11 bands of denticles in a distinct order (Fig 1A). Each band has a certain number of spots, for example, 46 spots in length and 5 spots in width for the seventh abdominal band. This pattern was dramatically changed in both zpl and zip mutants. The spots in the seventh abdominal band were reduced nearly by half (25-27 spots in length and 2-3 spots in width, Fig $1 \mathrm{~B}$

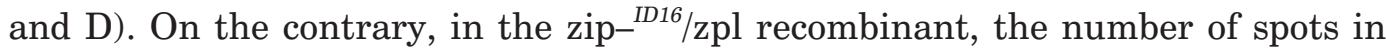
each bands increased, as compared with its parents (data not shown).

\section{The neural phenotype}

Specific staining of the nervous system in zpl mutant embryos showed a big brain and sometimes an enlarged thoracic region (data not presented here), which appeared after germ band shortening (stage 12), much later than the overgrowth of nervous system in neurogenic gene mutants[15]. Both of these abnormalities seemed to be due to the overgrowth of neural cells and axons in both region, as shown in Fig 2B and D. Just around the frontal commissure in mutant embryos, 


\section{Drosophila zpl gene for neurogenesis}

many newly formed cells were stained in the supraoesophageal region which must be derived from some neural cells as recognized by MAb-22C10, when compared with normal embryos as shown in Fig $2 \mathrm{~A}$ and $\mathrm{B}[16]$. There appeared some other unknown abnormal structures in the brain. They consisted of two groups of neural cells, one before and another behind the supraoesophague, and a bundle of neural fibres in between (Fig 2B). The same was true for zip mutant embryos[16]. Whether these abnormal, unknown structures were relevant to the loss of the hypophysis (Fig 2B) is still not clear. Nevertheless, the data above suggested that the zpl product is necessary for the maintain enance of normal growth of neural cells and axons.

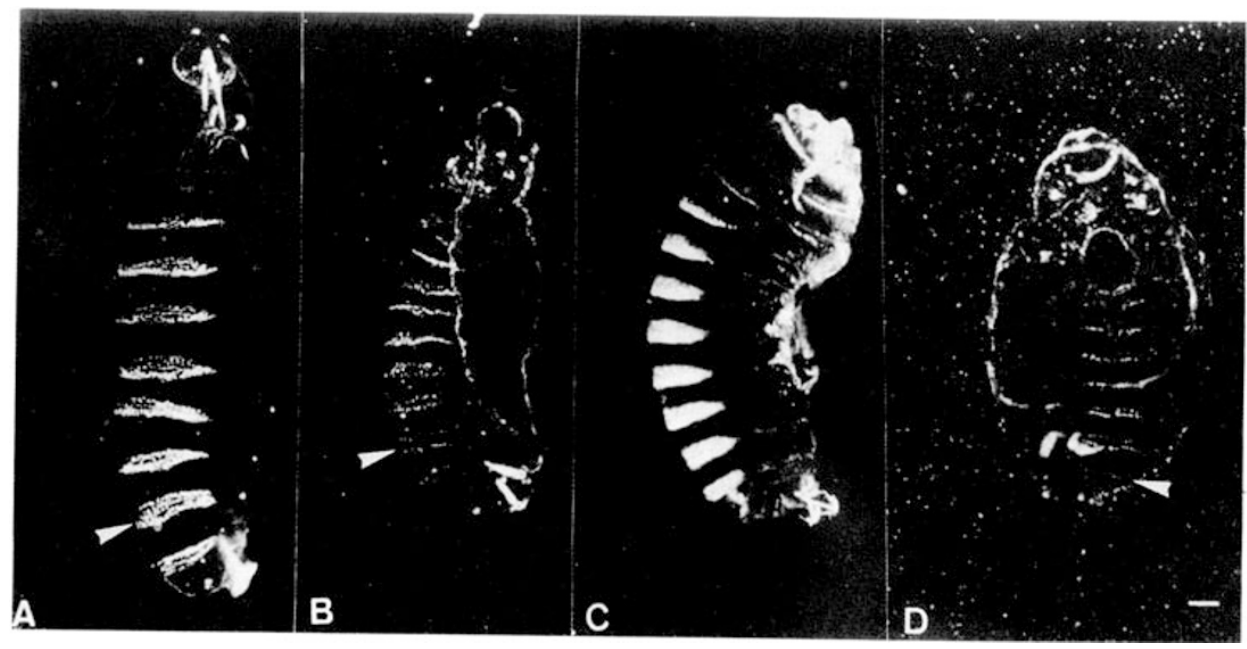

Fig 1. Cuticular phenotypes of zpl and zip mutant embryos

The cuticle of normal embryo was shown in A. zpl (B) and zip (D, a strong allele) mutant embryos had big holes from the head towards the abdomen. The weak zip mutant embryos (C) had small holes in both head and abdomen. The pattern of denticle rows in zpl (B) and strong zip mutant (D) obviously changed when compared with that of wild type embryo (A), especially the seven abdominal bands indicated by arrow heads. $(\times 200$, the bar $=5 \mathrm{~mm})$

After staining with anti-HRP antibodies or with 22C10 MAbs, the short and wrong pathway of fascicles in zpl mutant embryos were well visible (Fig 2D and F). In normal embryos, the growth cones of some fascicles from the CNS grew out from the ventral toward the lateral, and finally toward the dorsal direction until they nearly reached the dorsal midline (Fig $2 \mathrm{C}$ and E). But in zpl and strong zip mutants, they reached only the lateral regions, showing a wavy pattern (Fig 2D). The fascicles between different segmentation often contacted with each other, suggesting that some fascicles might have followed the wrong pathway, and that two or more fascicles from the PNS shared the same connection with the CNS (Fig 2F). Such wrong pathway could take different directions, as if the fascicle has lost its ability 
to recognize its surroundings at times and moved at random as a result of the fault of $\mathrm{zpl}$ gene product.

Fig 2. Aberrant neural system in the mutant embryos

Embryos in A, B, E and F were stained with monoclonal antibodies, 22C10. Embryos in $\mathrm{C}$ and $\mathrm{D}$ were stained with antibodies against HRP. A, C and $\mathrm{E}$ showed the normal embryos. B and D showed the overgrowth of neural cells and axons in the enlarged brain of zpl (or zip) mutant embryos as pointed by small arrow. Big arrow in B indicated the loss of the hypophysis as presented in the corresponding area in A. Arrow head in $B$ indicated two groups of newly formed cells connected by a bundle of neural fibres in supraesophageal region. $\mathrm{D}$ and $\mathrm{F}$ showed the short and wrong pathway of peripheral fascicles (arrow heads) in zpl (or zip) mutant embryos. $(\times 200-400$, bar $=5 \mathrm{~mm}$ )

\section{Complementation test}

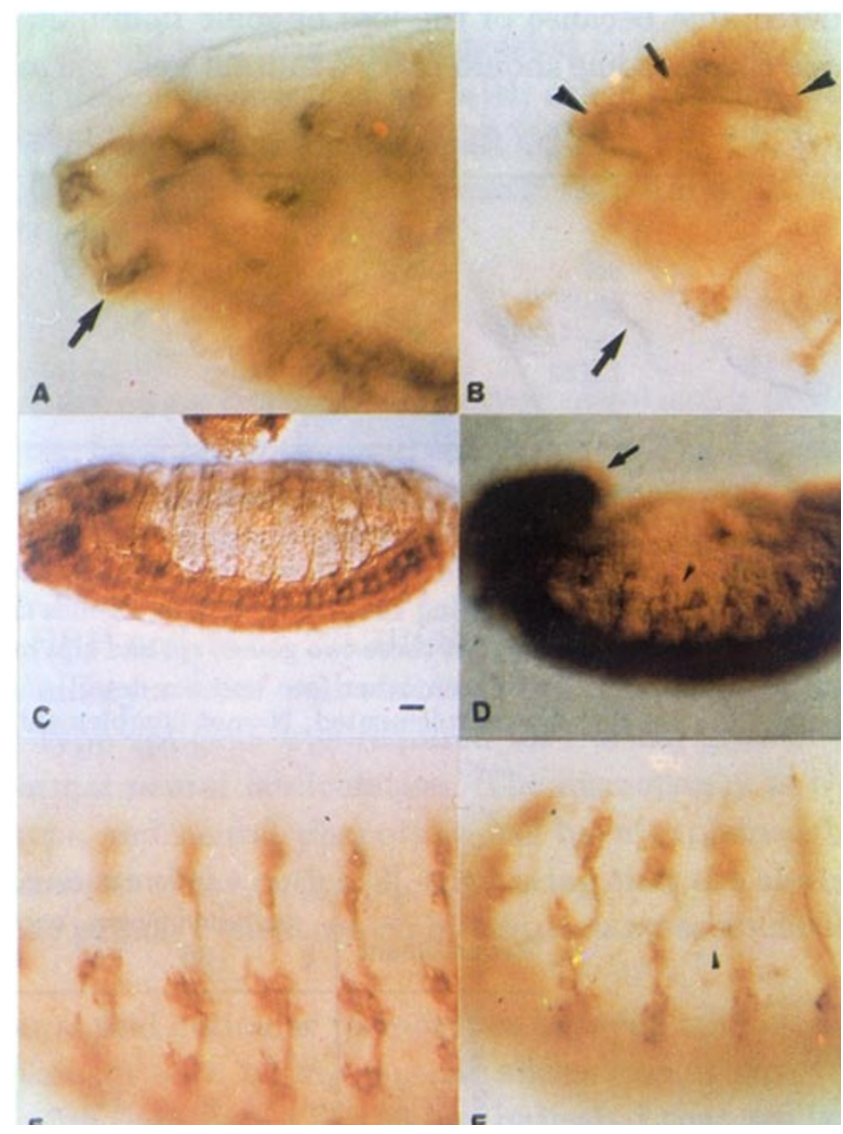

The cuticular and neural phenotype of zpl mutant embryos were exactly the same as those of zip mutants, especially the strong allele ID16, as described above and elsewhere[16]. The data summarized in Tab 2 showed that four zip mutants could not complement with each other, indicating that they were allele. However, zpl could complement with all zip mutants except zip ${ }^{I D 16}$, a strong zip point mutation. This suggests that zpl and zip are two different genes which are involved in normal neurogenesis.

\section{Preliminary genetic localization of zpl gene}

The finding of zpl mutant gene was resulted from the cleaning of the second chromosome background on the basis of the cuticular phenotype. Therefore, one specific mutant with five dominant markers on the second chromosome was used for an approximate localization of the zpl gene. The data in Fig 3A showed that all flies with straight wings, no matter whether they were recombinant or not, had the Pu2 


\section{Drosophila zpl gene for neurogenesis}

marker, in contrast to all other markers which were not consistently found in the population. From a genetic point of view, the recombinant flies, which were easily detectable because of the loss of some dominant markers, should be heterozygous: one chromosome should carry a mutant gene zpl and the other should carry dominant

Tab 2. Matrix of complementation tests within zip and zpl mutants

\begin{tabular}{lccccc}
\hline & & & & & \\
$\operatorname{zpl}$ & $\mathrm{zpl}$ & $\operatorname{zip}^{I D 16}$ & $\operatorname{zip}^{I I F 107}$ & IIX62 & ES1 \\
$\operatorname{zip}^{I D 16}$ & $\mathrm{~N}$ & $\mathrm{~N}$ & $\mathrm{~N}$ & $\mathrm{Y}$ & $\mathrm{Y}$ \\
$\operatorname{zip}^{I F 107}$ & $\mathrm{Y}$ & $\mathrm{N}$ & $\mathrm{N}$ & $\mathrm{N}$ & $\mathrm{N}$ \\
IIX62 & $\mathrm{Y}$ & $\mathrm{N}$ & $\mathrm{N}$ & $\mathrm{N}$ & $\mathrm{N}$ \\
$\mathrm{ES} 1$ & $\mathrm{Y}$ & $\mathrm{N}$ & $\mathrm{N}$ & $\mathrm{N}$ & $\mathrm{N}$ \\
& & & $\mathrm{N}$ & $\mathrm{N}$ \\
\hline
\end{tabular}

Note A. IIX62 and ES1 cannot complement with each other, because of the same part of the deficiency around zip and other genes.

B. zpl could complement with all other mutants tested except $\operatorname{zip}^{I D 16}$, suggesting that there were two genes causing a same zip phenotype and that these two genes,zpl and zip,might have a close relationship with each other (see text for detail). $\mathrm{Y}=$ complemented, $\mathrm{N}=$ not complemented.

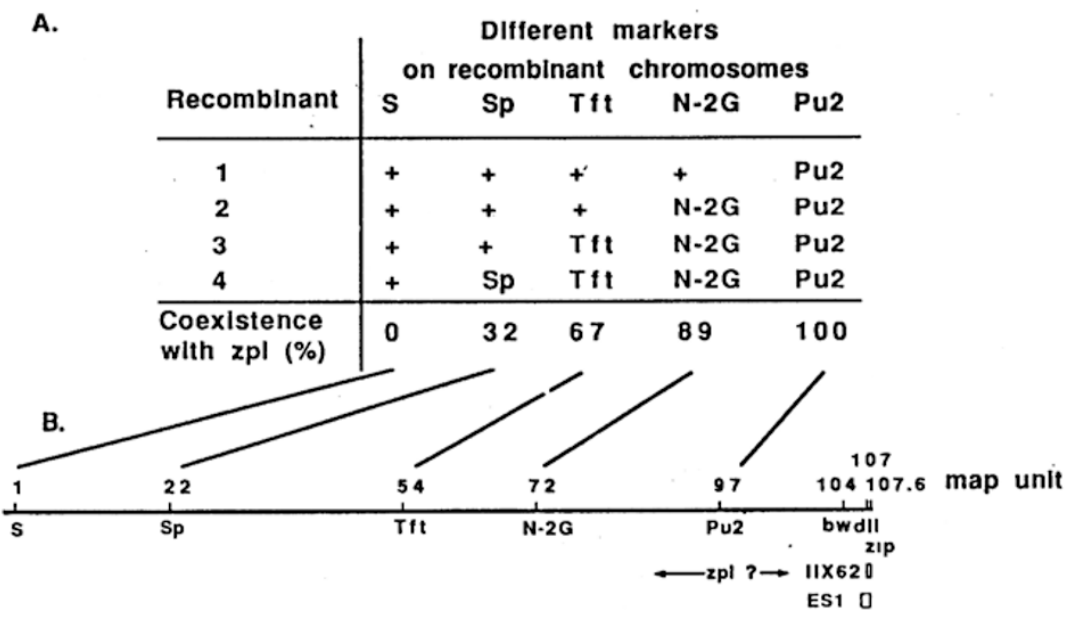

Fig 3. Genetic localization of zpl gene

A. Data from crosses between zpl and a mutant with several dominant markers. All flies with straight wing in F2 contained only the Pu2 marker, implying that zpl is near Pu2.

B. Rough genetic map of the zpl gene.

Note a. IIX62 and ES1 have a deficiency including zip and could rescue zpl; b. As the zpl wild type gene was always found together with $\mathrm{Pu} 2$, it seemed to be localized near the Pu2 marker (between Tft-2G and the proximal end of ES1 deficiency). 
markers and a wild type zpl or a mutant zpl gene. Since the recombinant second chromosome with the zpl mutant gene would have a lethal effect, all recombinant flies with straight wings must contain the recombinant chromosome with a wild type zpl gene. Thus the close linkage of zpl and Pu2 (Fig 3A), suggested that the mutant flies should carry both markers. The coexpression of zpl with other markers was $89 \%(\mathrm{~N}-2 \mathrm{G}), 67 \%$ (Tft) and $32 \%(\mathrm{Sp})$ as expected (Fig 3A). From Fig 3A, it is evident that the $\mathrm{zpl}$ gene is located near Pu2 (within the genetic map units 72-106, Fig 3B).

\section{DISCUSSION}

zpl gene was originally identified based on the same phenotype of cuticular defect as that of zip mutants. Failure of the head involution and the dorsal closure resulted in the cuticle hole (Tab 1). The specific staining of the nervous system with antibodies, however, has shown that the big brain in zpl and zip mutant embryos was due to the overgrowth of neural cells and axons (Fig 2B). In addition, after strong staining with antibodies against HRP and MAb-22C10, the short and misrouting of fascicles in zpl and zip mutant embryos were well visible (Fig 2D and F). It was obvious that the product of wild type zpl gene was required for normal growth of neural cells and axons, and for normal neural fasciculation. The discrepancy in the defects between cuticular phenotype and neural phenotype has been explained by the expression of zip in the CNS instead of the cuticle[9], suggesting that the abnormalities in nervous system were the primary effect which in turn caused secondarily the cuticular defect.

In spite of the similarity of neural and cuticular phenotypes between zpl and zip mutant embryos, zpl could complement with all zip mutant alleles except zip ${ }^{I D 16}$, a strong allele. The complementation data here (Tab 2) has indicated that $\mathrm{zpl}$ is a new gene, different from zip, which has been comfirmed by further genetic analysis, although we cannot find out the relation between these two genes at the moment.

$\mathrm{zpl}$ is located, like zip gene, on the second chromosome. Recombinant test of $\mathrm{zpl}$ with five dominant markers demonstrated that $\mathrm{zpl}$ was located in a different position than zip. Fig 3 A showed that all recombinant flies with straight wings had the Pu2 marker, but not other markers which were not consistently found in the population. Thus the $100 \%$ coexistence of $\mathrm{zpl}$ and Pu2 suggested that the mutant flies should carry both genes. Given the zpl gene was located just near Pu2 (about 75 map units on the second chromosome, Fig 3B), the coexistence of $\mathrm{zpl}$ with other markers was $89 \%(\mathrm{~N}-2 \mathrm{G}), 67 \%(\mathrm{Tft})$ and $32 \%(\mathrm{Sp})$ as expected.

All above data hinted to the fact that $\mathrm{zpl}$ and zip were not the same gene, even though the $\mathrm{zpl}$ gene mutant showed exactly the same phenotype as zip. It is, however, most likely that there is a close relation between the products of $\mathrm{zpl}$ and zip genes, although it is still not clear why zpl cannot be complemented by one of the zip mutants, $\operatorname{zip}^{1 D 16}$, at present time. If a dimer-like protein complex is formed 


\section{Drosophila zpl gene for neurogenesis}

between the products of zpl and zip genes[16], the mutation of one of these two genes would naturally cause the same phenotype during embryogenesis, as shown by zpl or zip mutant embryos. And uncomplementation between zpl and zip ${ }^{-I D 16}$, a strong zip mutant, could possibly be explained by the insufficiency of one fourth normal zip-zpl complexes in heterozygous embryos.

\section{ACKNOWLEDGEMENT}

We would like to thank our colleagues in Dr. H. Jaeckle's laboratory for many stimulating discussions during the course of the work, Dr. Nusslein-Volhard C. for the use of zip mutant flies and Dr. A. Ferrus for the gift of monoclonal antibodies. Special thanks to Dr. W. Hennig for critical reading and comments on the manuscript. D. Zhao was supported by the Ministry of Education of China, and by a Max-Planck-Society Fellowship. The work was supported by a DFG grant (Leibniz-programm) and SJLLS.

\section{REFERENCES}

[1] Compos-Ortega JA, Hartenstein V. The Embryonic Development of Drosophila melanogaster. Springer Verlag Berlin-Heidelberg 1985.

[2] Zhao D, et al. Developmental distribution of zip protein revealed by antibodies against zip gene products. Acta Biol Exper Sinica 1994; in press.

[3] Brand M, Campos-Ortega JA. Two groups of interrelated genes regulate early neurogenesis in Drosophila melanogaster. Roux' Arch Dev Biol 1988; 197:457-70.

[4] Doe CQ, Scott MP. Segmentation and homeotic gene functioin in the developing nervous system of Drosophila. TINS 1988; 11:101-6.

[5] Compos-Ortega JA, Jan YN. Genetic and molecular bases of neurogenesis in Drosophila melanogaster. Annu Rev Neurosci 1991; 14:399-420.

[6] Jan Y, Jan LY. Genes required for specifying cell fates in Drosophila embryonic sensory nervous system. TINS 1990; 13:493-8.

[7] Steller H, Fischbach K, Rubin GM. disconnected: A locus required for neural pathway formation in the visual system of Drosophila. Cell 1987; 50:1139-53.

[8] Patel NH Snow PM, Goodman CS. Characterization and cloning of fasciclin III: a glycoprotein expresssed on a subset of neurons and axon pathways in Drosphila. Cell 1987; 48:975-88.

[9] Zhao D, Cote S, Jaehnig F, Haller J, Jaeckle H. zipper encodes a putative integral membrane protein required for normal axon patterning during Drosophila neurogenesis. The EMBO J 1988; 7:1115-9.

[10] Cote S, Preiss A, Haller J, et al. The gooseberry-zipper region of Drosophila: five genes encode different spatially restricted transcripts in the embryo. EMBO J. 1987; 6:2793-801.

[11] Nuesslein-Volhard C, Wieschaus E, Kluding H. Mutation affecting the pattern of the larval cuticle in Drosohila melanogaster. I. Zygotic loci on the second chromosome. Roux' Arch Dev Biol 1984; 193:267-82.

[12] Jan LY, Jan YN. Antibodies to horseadish peroxidse as specific neural markers in Drosophila and in grasshopper. PNAS USA 1982; 72:2700-4.

[13] Zipursky SL, Venkatesh TR, Teplow B, Benzer R. Neuronal development in the Drosophila retina: Monoclonal antibodies as molecular probes. Cell 1984; 36:15-26.

[14] Gaul U, Seifert E, Schuh R, Jaeckle H. Analysis of Kruppel protein distribution during early Drosophila development reveals posttranscriptional regulation. Cell 1987; 50:639-47. 
[15] Lehmann B,, Jimenez F, Dietrich U, Campos-Ortega JA. On the phenotype and development of mutants of early neurogenesis in Drosophila melanogaster. Roux' Arch Dev Biol 1983; 192:6274.

[16] Zhao D. Genetic and Molecular Analysis of zip Gene in Drosophila. Ph.D. Dissetation. Academia Sinica 1988.

Received 23-4-1994. Revised 25-8-1994. Accepted 29-8-1994. 